\title{
Y984028 火星陨石的岩石矿物学特征和冲击变质
}

\author{
胡森 ${ }^{(12)}$, 冯珵畧 ${ }^{(2)}$, 林杨挺 ${ }^{(1 *}$ \\ (1) 中国科学院地质与地球物理研究所地球深部结构与过程研究室, 北京 100029 ; \\ (2) 中国科学院研究生院, 北京 100049 \\ * 联系人, E-mail: linyt@mail.igcas.ac.cn \\ 2011-01-26 收稿, 2011-02-17 接受 \\ 中国科学院知识创新工程资助项目(KZCX2-YW-110, KZCX2-YW-Q08)
}

\begin{abstract}
摘要 Y984028 是一块新的二辉橄榄岩质火星陨石, 具有两种明显不同的岩石结构: 粒间结构 和嵌晶结构. 与嵌晶结构区域的矿物相比, 粒间区域的铁镁质硅酸盐贫 $\mathrm{FeO}$ 、铬铁矿贫 $\mathrm{TiO}_{2}$. 虽 然Y984028 的岩石结构和矿物化学成分与 GRV 99027 非常相似, 但两者的冲击变质特征有明显 差异, 表明 Y 984028 不是 GRV 99027 的成对陨石. Y 984028 经历了强烈的冲击作用, 冲击后由于 该陨石没有遭受明显的热变质, 因而该样品是研究类地行星早期撞击过程的探针. Y 984028 熔脉 中主要包含细小的矿物碎屑, 部分矿物边缘发育细粒化结构, 没有发现任何高压相矿物, 表明 Y984028 在撞击过程中发生了部分熔融, 在较低压力下发生淬火.
\end{abstract}

关键词

火星陨石

二辉橄榄岩

南极

冲击变质
二辉橄榄岩质火星陨石是火星陨石的一个小群, 至今只有 12 块该类型的陨石. 所有的二辉橄榄岩质 火星陨石都由两种明显不同的岩石结构组成: 嵌晶 结构和粒间(非嵌晶)结构. 嵌晶结构主要由易变辉石 主晶和橄榄石客晶组成, 辉石和橄榄石颗粒内有少 量自形铬铁矿包裹体. 粒间结构主要由自形-半自形 橄榄石、普通辉石、易变辉石和粒间充填的长石玻璃 组成, 铬铁矿、钛铁矿、磁黄铁矿和磷酸盐等次要矿 物与长石玻璃共生. 此外, 二辉橄榄岩质火星陨石的 矿物化学成分呈现双峰分布: 粒间区域的橄榄石和 辉石富 $\mathrm{FeO}$ 、铬铁矿富 $\mathrm{TiO}_{2}$, 嵌晶区域的橄榄石和辉 石相对贫 $\mathrm{FeO}$ 、铬铁矿贫 $\mathrm{TiO}_{2}$.

前人对 3 块二辉橄榄岩质火星陨石 (Allan Hill (ALH) A77005, Yamato (Y-) 793605 和 Lewis Cliff (LEW) 88516)进行了大量研究, 结果发现该类陨石 均有相似的岩石结构和矿物成分 ${ }^{[16]} 、 \mathrm{Sm}-\mathrm{Nd}$ 和 $\mathrm{Rb}-$ $\mathrm{Sr}$ 等时线年龄 ${ }^{[7,8]}$ 和溅射年龄 ${ }^{[9 ~ 11]}$. 这表明二辉橄榄 岩质火星陨石可能来自火星的同一岩浆单元, 并在 同一次撞击事件中离开火星母体. 近年来, 在南极和 沙漠地区发现了较多的二辉橄榄岩质火星陨石, 如
GRV 99027 $7^{[12 \sim 15]}$, Yamato (Y)000027(与 Y000047 和 Y000097 成对 $)^{[16]}$ 和 NWA $1950^{[17]}$, 这些新发现的二 辉橄榄岩质火星陨石也具有相似的岩石结构、矿物成 分、 $\mathrm{Sm}-\mathrm{Nd}$ 和 $\mathrm{Rb}-\mathrm{Sr}$ 等时线年龄和溅射年龄. 只有 GRV 020090 ${ }^{[14,18]}$ 和 Roberts Massif (RBT) 04261 (与 RBT 04262 成对) ${ }^{[19,20]}$ 的稀土含量和不相容元素明显 偏高, 可能来自岩浆房的顶部或富集的火星幔. 对新 发现的二辉橄榄岩质火星陨石进行研究可对该族陨 石的岩石学成因提供重要补充.

\section{1 样品和实验条件}

Y984028 是一块新发现的二辉橄榄岩质火星陨 石(Meteorite Newsletter 17, 2008). 为澄清该陨石与 其他同类陨石之间的联系, 日本极地研究所 Misawa 博士组织了一个小型国际合作研究. 作为该合作项 目的一部分, 我们主要负责 Y984028 的岩石学和矿 物学的工作, 与我国的火星陨石 GRV 99027 和其他 二辉橄榄岩质火星陨石进行对比研究. 最初的研究 成果可见文献[21].

本文使用的样品为光薄片, 编号 Y984028 51-2.

英文版见: Hu S, Feng L, Lin Y T. Petrography, mineral chemistry and shock metamorphism of Yamato 984028 lherzolitic shergottite. Chinese Sci Bull, 2011, 56, doi: 10.1007/s11434-011-4443-x 
该光薄片中可见黑色的冲击熔脉贯穿整个薄片, 在 四边形样品的一侧残留少量熔壳. 该薄片的岩石学 结构在光学显微镜和扫描电子显微镜下完成. 扫描 电子显微镜为中国科学院地质与地球物理研究所的 LEO $1450 \mathrm{VP}$, 配有牛津 INCA X 射线能谱仪, 可对矿 物成分进行半定量分析, 加速电压为 $15 \mathrm{kV}$. 拉曼光 谱测试在中国科学院理化技术研究所的显微共焦激 光拉曼光谱仪 in-via reflex Renishaw 上完成, 激光光 源为 $\mathrm{Ar}^{+}$, 波长 $532 \mathrm{~nm}$, 功率为 $16 \mathrm{~mW}$, 实际作用在 样品表面的束斑为 $1 \mu \mathrm{m}$. 矿物化学成分定量分析在 中国科学院地质与地球物理研究所的电子探针 JEOL JXA8100 上完成, 分析条件为: 加速电压 $15 \mathrm{kV}$, 电 子束流 $20 \mathrm{nA}$ (长石 $10 \mathrm{nA}$ ). 橄榄石、辉石和金属采 用聚焦束斑, 长石则采用非聚焦束斑 $(10 \mu \mathrm{m})$, 以避 免 $\mathrm{Na}$ 等元素的挥发丢失. 硅酸盐矿物定量分析的标 准包括自然产出的和合成的矿物: $\mathrm{Si}, \mathrm{Mg}$ 和 $\mathrm{Fe}$ 的分 析标准为角闪石, $\mathrm{Na}$ 和 $\mathrm{Al}$ 的分析标准为硬玉, $\mathrm{K}$ 的标 准为钾长石, $\mathrm{Ca}$ 和 $\mathrm{Mn}$ 的分析标准为钙萻薇辉石, $\mathrm{Ti}$ 的标准为金红石, $\mathrm{Cr}$ 的标准为 $\mathrm{Cr}_{2} \mathrm{O}_{3}$. 分析过程中, 对一些元素特征峰的叠加进行了校正, 如 $\mathrm{Cr}$ 的 $\mathrm{K}_{\beta}$ 线 对 $\mathrm{Mn}$ 的 $\mathrm{K}$ 线的叠加. 元素的分析检出限为 $(1 \sigma$, $w t \%): \mathrm{K}_{2} \mathrm{O}$ 为 $0.01 ; \mathrm{SiO}_{2}, \mathrm{Al}_{2} \mathrm{O}_{3}, \mathrm{MgO}, \mathrm{CaO}$ 和 $\mathrm{Na}_{2} \mathrm{O}$ 为 $0.02 ; \mathrm{TiO}_{2}$ 和 $\mathrm{Cr}_{2} \mathrm{O}_{3}$ 为 $0.03 ; \mathrm{FeO}$ 为 $0.05 ; \mathrm{MnO}$ 为 0.06 .
硅酸盐矿物的分析结果采用 Bence-Albee 方法计算. 矿物模式含量主要根据背散射电子图像上灰度差异 来估算, 并归一到 $100 \mathrm{vol} \%$ (vol\%体积百分, 余同).

\section{2 结果}

\section{1 岩石学特征}

Y984028 有两种不同的岩石结构(图 1), 即嵌晶 结构和粒间结构 (有些学者称之非嵌晶结构), 为二辉 橄榄岩质火星陨石的典型特征. 在嵌晶区域, 浑圆状 橄榄石客晶(50 1200 $\mu \mathrm{m}, 39.6 \mathrm{vol} \%)$ 与细粒自形铬铁 矿 (30 100 $\mu \mathrm{m}, 1.1 \mathrm{vol} \%)$ 被易变辉石主晶包裹 $(58.3$ $\mathrm{vol} \%)$; 粗粒的易变辉石主晶边缘常有一层普通辉石 薄边. 粒间区域主要由自形-半自形的橄榄石 (68.2 $\mathrm{vol} \%$ )、辉石(15.2 vol\%)和长石(15.1 vol\%)组成. 粒间 区域中的橄榄石与辉石的含量比(4.5)明显高于嵌晶 区域 (0.7). 富 $\mathrm{Ti}$ 铬铁矿 (1.1 vol\%) 和磁黄铁矿 $(0.4$ vol\%)仅产出于粒间区域, 主要位于长石玻璃的边部. 在这两种结构中, 橄榄石在透光下均呈淡黄色, 辉石 没有出熔. 在正交偏光下可见长石全消光, 已经转变 成玻璃, 拉曼光谱也显示为玻璃相. Y984028 的模式 含量见表 1 .

在高对比度背散射电子图像上, 可见明暗两种

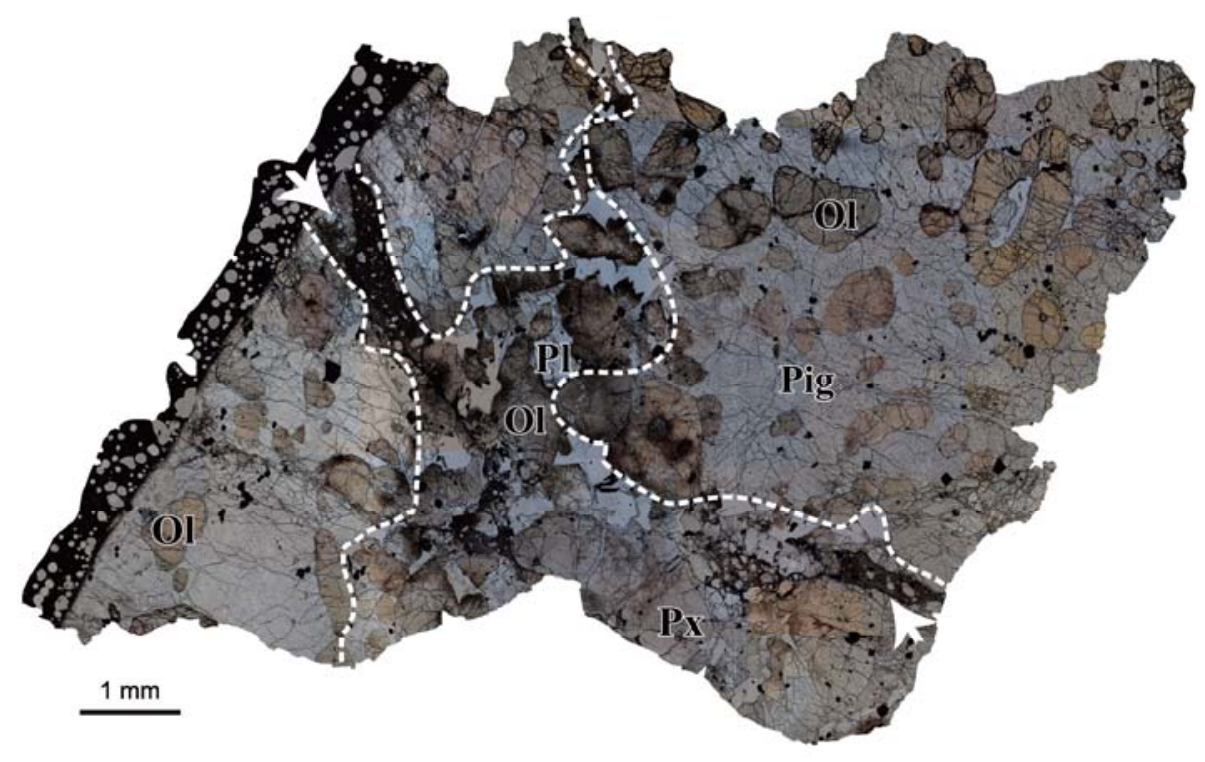

图 1 Y984028 透光图

中央部分为粒间结构(虚线圈出部分), 主要由橄榄石 $(\mathrm{Ol})$ 、辉石 $(\mathrm{Px})$ 和粒间长石 $(\mathrm{Pl})$ 组成. 其余部分为嵌晶区域, 主要由橄榄石客晶和易 变辉石 $(\mathrm{Pig})$ 主晶组成. 被橄榄石和辉石包裹的细小的黑色自形矿物为铬铁矿. 薄片上可见冲击熔脉(箭头所指)和最左边的熔壳. 橄榄石 在透光下呈淡黄色 
表 1 Y984028 模式含量与其他二辉橄榄岩质火星陨石对比(vol\%)

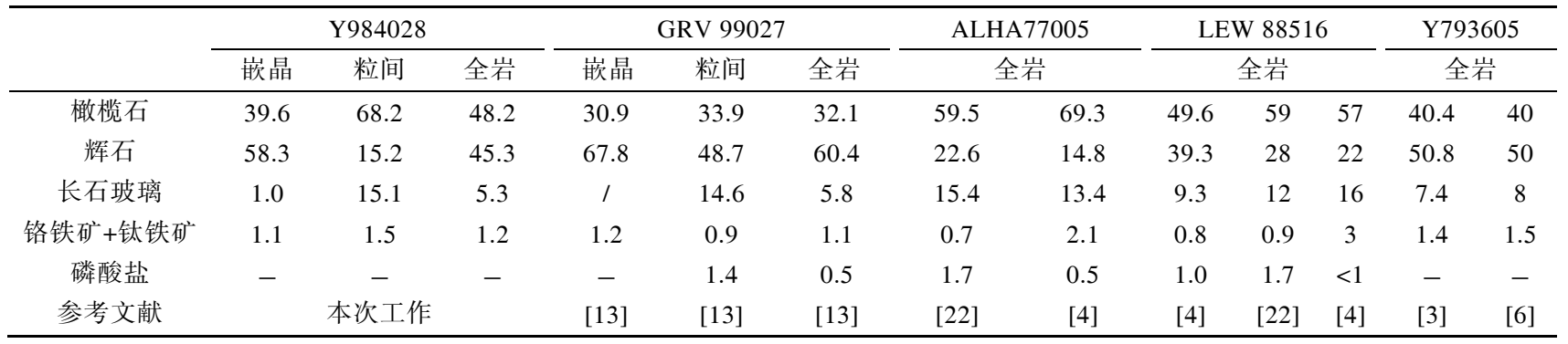

“_”, 磷酸盐含量累计在辉石含量中; “””, 几乎没有

长石玻璃共生(图 2(a)和(b)). 较暗的长石玻璃常产出 于亮长石玻璃的边部或粒间. 在长石包裹的矿物角 砾中可见细粒的辉石、白磷钙矿、钛铁矿与次生斜锆 石共生(图 2(c)), 这些矿物可能在结晶的晚期形成. 少许斜锆石 $(<5 \mu \mathrm{m})$ 与钛铁矿共生. 磁黄铁矿中可见 富 Ni 的针状颗粒, 可能是镍黄铁矿 (图 2(d)).

Y984028 中可见大量岩浆包裹体. 橄榄石中岩 浆包裹体数量最多, 包裹体直径可达 $120 \mu \mathrm{m}$. 相比, 辉石和铬铁矿中岩浆包裹体数量较少(图 3). 不同矿 物中的岩浆包体不仅数量和粒度有差异, 在岩石学
上也有较大差别，橄榄石中的岩浆包裹体呈球状或 卵状, 主要由长石质玻璃和辉石组成, 辉石为板状, 表现出成分环带特征，被长石质玻璃包裹，长石质玻 璃中还常见石英质玻璃, 橄榄石与岩浆包裹体之间 常有一层辉石边 (图 3(a) 和 (c)), 为富 $\mathrm{Ca}$ 辉石. 在嵌晶 区域的橄榄石中，有些岩浆包裹体包含富钙和贫钙 两种辉石、富钾长石和少量尖晶石, 该包裹体全部重 结晶(图 3(b)). 铬铁矿中的岩浆包裹体粒度最小 $(<5$ $\mu \mathrm{m})$, 呈圆球状, 与铬铁矿有清晰的边界, 主要由长 石玻璃、石英和磁黄铁矿组成(图 3(d)). 在所有包裹
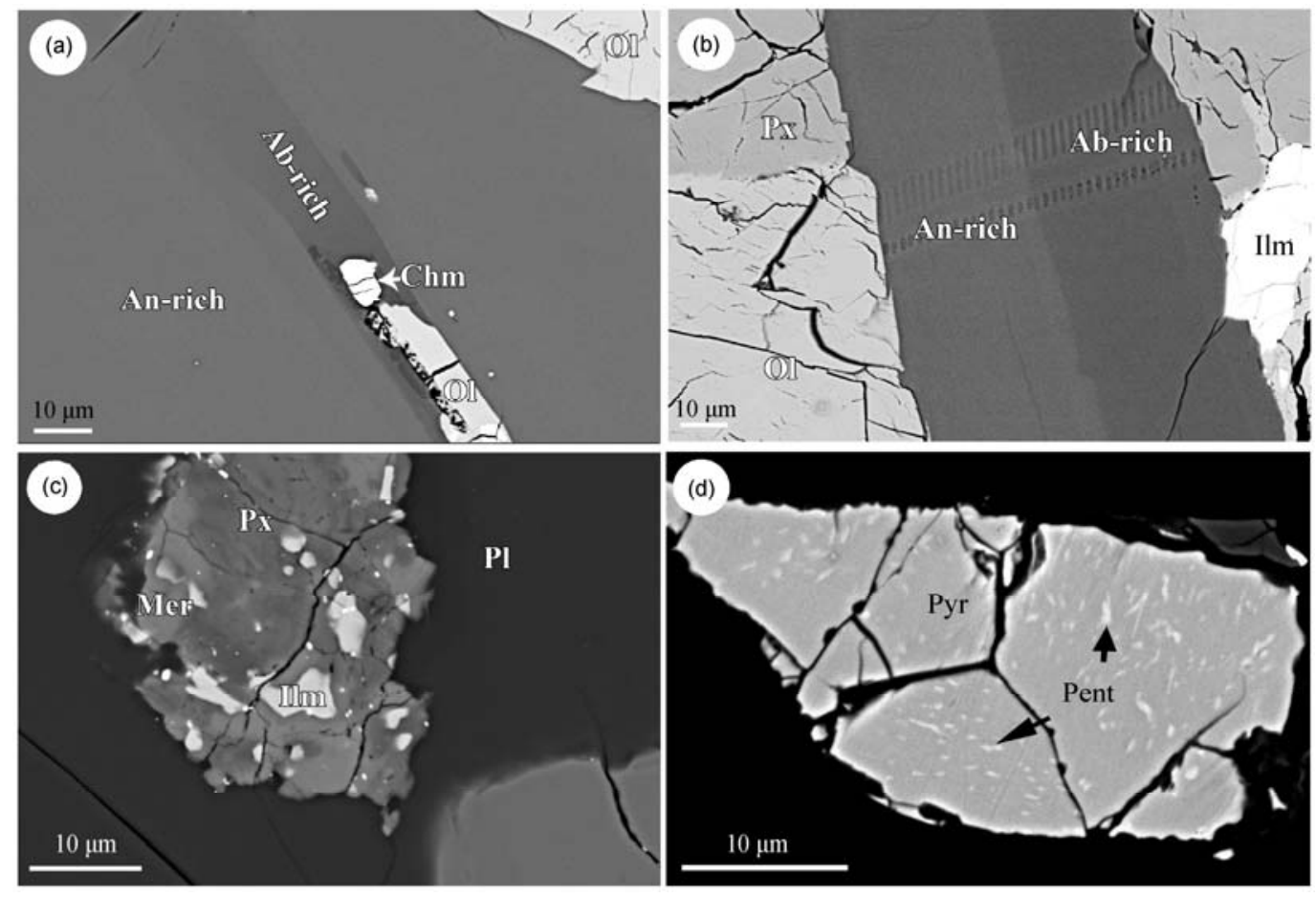

图 2 Y984028 背散射电子图像

(a) 富 $\mathrm{Na}$ 长石 $(\mathrm{Ab}-\mathrm{rich}) 、$ 铬铁矿 $(\mathrm{Chm})$ 和橄榄石 $(\mathrm{Ol})$ 被富 $\mathrm{Ca}$ 长石 $(\mathrm{An}-\mathrm{rich})$ 包裹; (b) 富 $\mathrm{Na}$ 长石与富 $\mathrm{Ca}$ 长石共生; (c) 长石中残留的矿物 集合体, 由辉石 $(\mathrm{Px})$ 、白磷钙矿 (Mer)、钛铁矿 (Ilm) 和少量斜锆石(白色斑点)组成; (d) 磁黄铁矿 (Pyr)中可见富 Ni 的针状颗粒, 可能是镍 黄铁矿(Pent) 


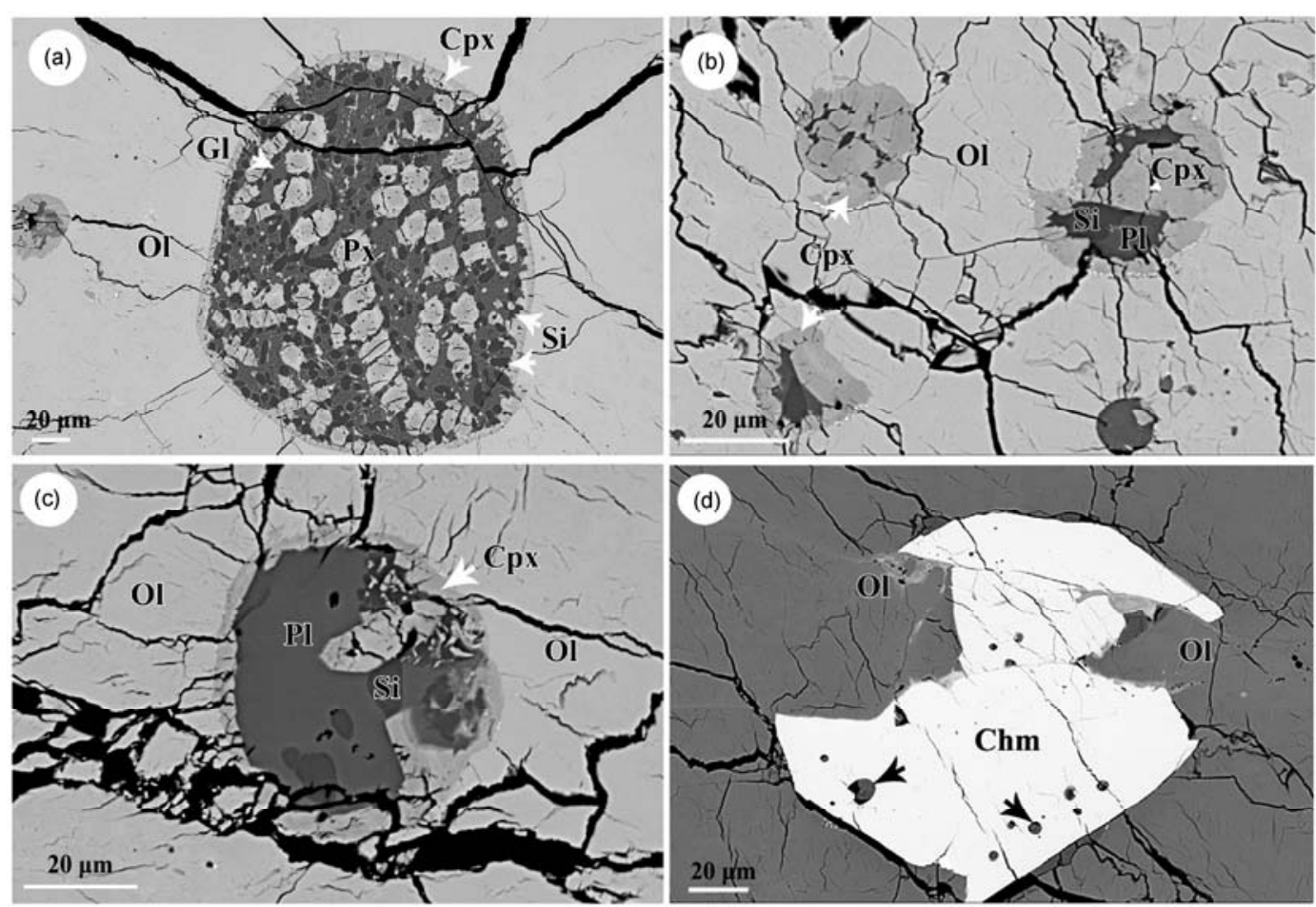

图 3 Y984028 岩浆包裹体的背散射电子图像

(a) 橄榄石中大的岩浆包裏体, 由富 $\mathrm{Ca}$ 辉石 $(\mathrm{Cpx})$ 、石英 $(\mathrm{Si})$ 和长石质玻璃 $(\mathrm{Gl})$ 组成; (b) 橄榄石中小的岩浆包裹体, 由富 $\mathrm{Ca}$ 辉石、石英 和长石质玻璃组成; (c) 橄榄石中的岩浆包裏体，可见富 $\mathrm{Ca}$ 辉石边; 自形石英被长石质玻璃包裏; (d) 铬铁矿 (Chm)中的岩浆包裏体

体中, 没有发现任何高压相矿物.

\section{2 冲击特征}

Y984028 经历过强烈的冲击变质, 所有的长石 已经转变成玻璃相. 橄榄石和辉石在正交偏光下普 遍存在马赛克消光. 在薄片中可见宽度约 $1 \mathrm{~mm}$ 的冲 击熔融脉(图 1). 熔脉中的基质主要由硅酸盐质玻璃 和细粒不规则状矿物碎屑组成, 其中长石质玻璃在 熔脉中呈弯曲或细长的条带, 表现出明显的流动结 构. 基质中可见大量粗颗粒硅酸盐角砾, 没有发现因 冲击作用形成的橄榄石、辉石和长石的高压多形(图 4(a) 和(b)). 有些角砾大部分已经细粒化, 部分颗粒 与基质的边界不清晰(图 4(b)和(c)). 有少许橄榄石细 颗粒边缘呈现重结晶现象，从核部到边部成韵律状 环带变化(图 4(d)). 熔脉中的铬铁矿常呈网状, 沿橄 榄石和辉石边部生长(图 4(b)).

Y984028 冲击熔脉的结构特征及矿物组合与普 通球粒陨石中的冲击熔脉有显著差异, 后者的基质 为高压下从熔体中结晶形成的自形-半自形细粒硅酸 盐质颗粒，其中的橄榄石、辉石以及长石角砾普遍在
峰值高压作用下转变为林伍德石、镁铁榴石和熔长石 等高压相 ${ }^{[23]}$. 而 Y984028 中的矿物形貌和结构特征 更可能是在较低压力下 (压力卸载阶段)、较高温度条 件下发生熔融形成.

除上文所述的冲击熔脉外, Y984028 中还有一些 因冲击形成的微型细脉, 脉宽只有 $10 \sim 30 \mu \mathrm{m}$. 这些 细脉的结构与粗脉有明显不同(图 5). 在辉石颗粒中 的细脉发育淬火形成的树枝状结晶, 细脉边缘的辉 石可见因部分熔融形成光滑均一的带状区域(图 5(a)). 橄榄石细脉中也可见部分熔融，然而并不发育枝状 结晶. 橄榄石颗粒与细脉边部接触区域可见 2 个方向 的出溶(图 5(a)和(b)).

拉曼光谱分析结果显示，冲击熔脉的细粒基质 和粗颗粒硅酸盐角砾中并未发现橄榄石、辉石和长石 的高压相特征吸收峰. 橄榄石细脉以及边部出溶区 域仅呈现典型的橄榄石拉曼吸收峰. 辉石颗粒中与 细脉边部接触的光滑区域(图 5(a))已经转变成玻璃.

\section{3 矿物成分}

橄榄石：Y984028 中橄榄石的成分与岩石结构具 

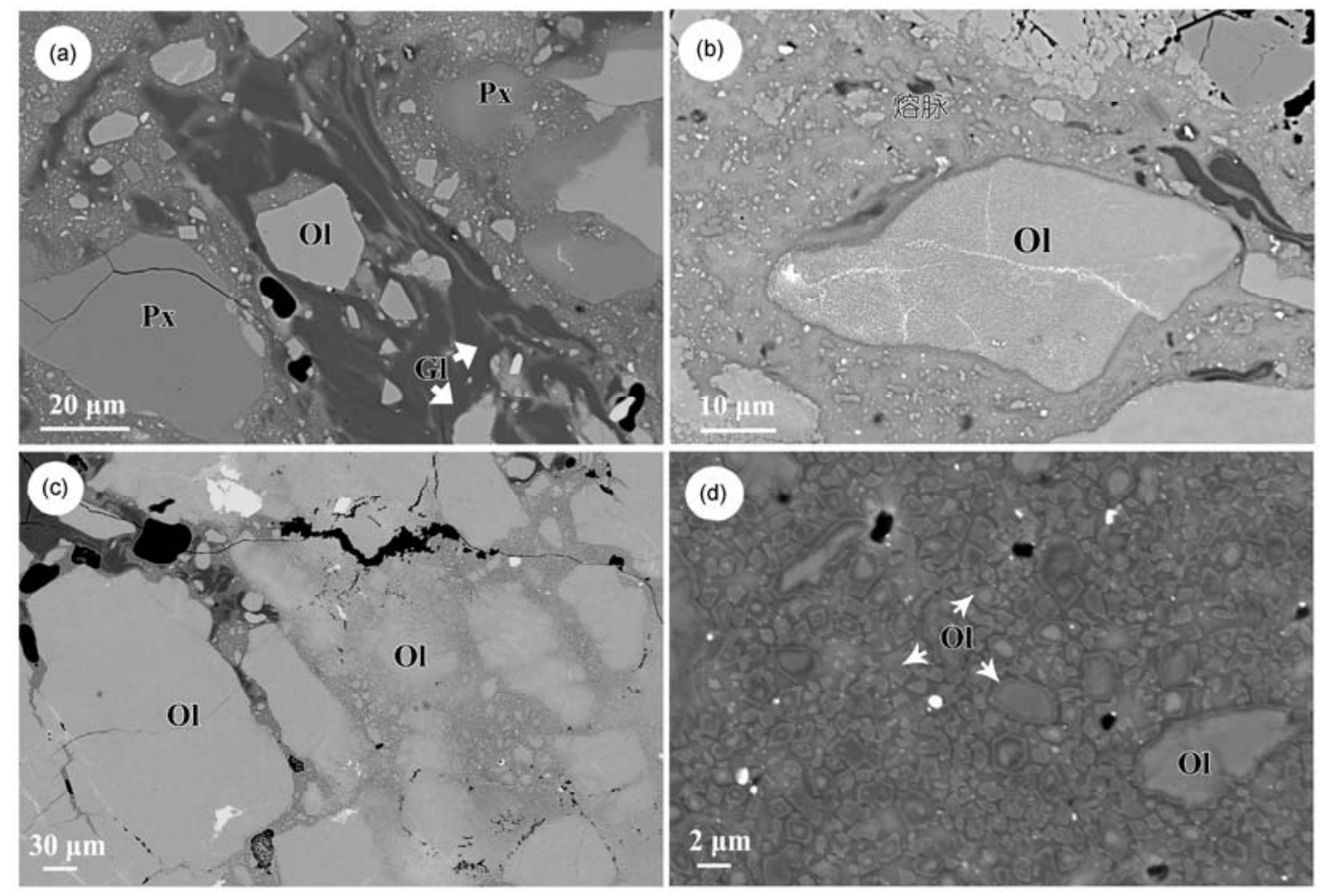

图 4 Y984028 中的冲击熔脉

(a) 熔脉中的橄榄石 $(\mathrm{Ol})$ 和辉石 $(\mathrm{Px})$, 熔脉中可见典型的流动结构, 主要为深黑色条带状长石玻漓 $(\mathrm{Gl})$ 组成, 长石玻璃间可见细带状橄榄 石、辉石熔体(亮灰色); (b) 熔脉中的橄榄石碎屑, 可见明显的破碎和冲击后熔融特征; (c) 熔脉中的粒状檄榄石因部分熔融, 边缘部分 为重结晶的细粒颗粒, 轮廓不清晰; (d) 熔脉中呈环带的橄榄石颗粒
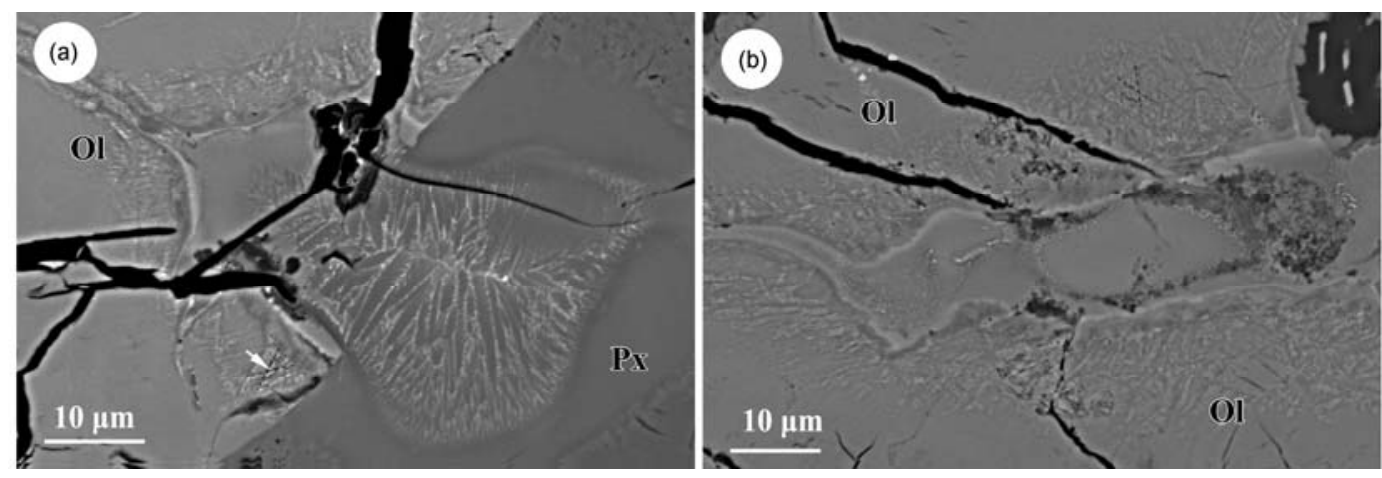

图 5 Y984028 中冲击形成的细熔脉

(a) 冲击细脉中的辉石 $(\mathrm{Px})$ 呈树枝状, 被光滑的辉石包裏, 细熔脉边部的橄榄石 $(\mathrm{Ol})$ 有两个方向的出溶条带; (b) 橄榄石颗粒中的细脉, 其中橄榄石 $(\mathrm{Ol})$ 也有 2 个方向的出溶条带

有相关性(图 6). 嵌晶区域的橄榄石 $\mathrm{FeO}$ 含量为 27.8 $\pm 1.5 \mathrm{~mol} \%$ (25.5 mol\% 30.8 mol\%(mol\%, 摩尔百分, 余同)), 明显低于粒间区域的 $\mathrm{FeO}$ 含量 $(31.4 \pm 0.9$ $\mathrm{mol} \%$, 29.6 33.5 mol\%). 电子探针数据显示两种结 构的橄榄石颗粒从核部到边部的化学成分非常均一 (表 2). 所有的橄榄石含少量 $\mathrm{MnO}(0.54 \pm 0.05 \mathrm{wt} \%)$ 和 $\mathrm{CaO}(0.18 \pm 0.06 \mathrm{wt} \%)$ (wt \% , 重量百分, 余同). 橄 榄石的 $\mathrm{FeO} / \mathrm{MnO}$ 质量比与岩石结构无关, 为 $49.1 \pm$

\section{2. 橄榄石代表性电子探针成分见表 2 .}

易变辉石: 与橄榄石相似, 易变辉石的成分也与 岩石结构有关(图 7 和 8). 与粒间区域的易变辉石 $\left(\mathrm{Fs}_{24.6 ~ 29.8}\right)$ 相比, 嵌晶区域的易变辉石 $\mathrm{FeO}$ 含量较低 $\left(\mathrm{Fs}_{21.423 .9}\right)$ (图 7). 易变辉石的次要元素与主量元素相 似，与岩石结构相关. 易变辉石在粒间区域的 $\mathrm{Al}_{2} \mathrm{O}_{3}$, $\mathrm{TiO}_{2}$ 和 $\mathrm{CaO}$ 含量高于嵌晶区域, 分别为 $0.64 \pm 0.36$ $\mathrm{wt} \%, 0.17 \pm 0.13 \mathrm{wt} \%$ 和 $3.15 \pm 0.98 \mathrm{wt} \%$ (粒间区域); 


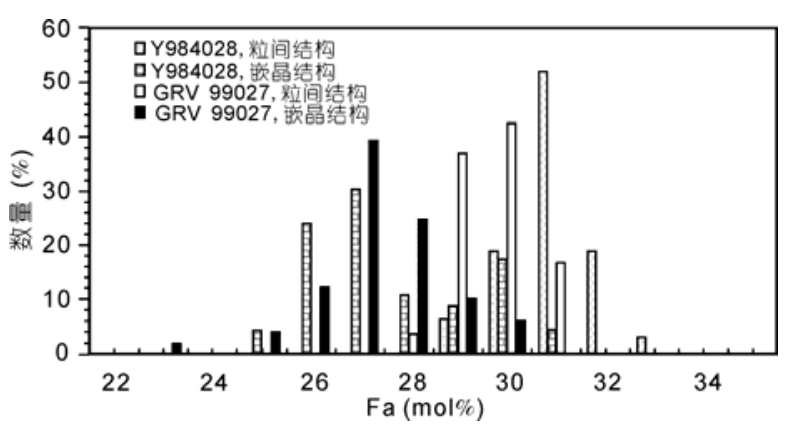

图 6 橄榄石 $\mathrm{Fa}$ 值频谱分布图

与嵌晶区域的橄榄石相比, Y984028 陨石粒间区域的橄榄石明显 富 $\mathrm{FeO}$, 与 GRV 99027 的橄榄石频谱分布非常相似, 嵌晶区域与 粒间区域的橄榄石成分差异比 GRV 99027 稍大 ${ }^{[13]}$

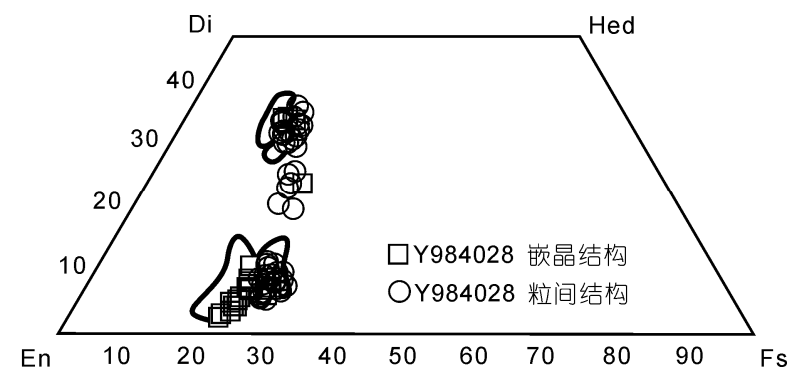

图 7 Y984028 中易变辉石和普通辉石的四端元组分图 嵌晶区域与粒间区域的易变辉石有明显的成分差异, 嵌晶区域易 变辉石相对贫 $\mathrm{FeO}$; 普通辉石几乎没有差异. 黑线圈出区域为 GRV 99027 辉石的成分分布范围 ${ }^{[13]}$

表 2 Y984028 主要和次要矿物代表性电子探针数据 ${ }^{*}(w \mathrm{t} \%)$

\begin{tabular}{|c|c|c|c|c|c|c|c|c|c|c|c|c|c|c|c|c|c|c|c|c|}
\hline & \multicolumn{10}{|c|}{ 粒间区域 } & \multicolumn{6}{|c|}{ 嵌晶区域 } & \multicolumn{4}{|c|}{ 岩浆包裹体 } \\
\hline & $\mathrm{Chm}^{\mathrm{a})}$ & $\mathrm{Ilm}^{\mathrm{b})}$ & $\mathrm{Ol}^{\mathrm{b})}$ & $\mathrm{Ol}^{\mathrm{a})}$ & $\mathrm{Pig}^{\mathrm{b})}$ & $\mathrm{Pig}^{\mathrm{a})}$ & $\mathrm{Aug}^{\mathrm{a})}$ & $\mathrm{Aug}^{\mathrm{a})}$ & $\mathrm{Pl}^{\mathrm{a})}$ & $\mathrm{Pl}^{\mathrm{a})}$ & $\mathrm{Chm}^{\mathrm{a})}$ & $\mathrm{Ol}^{\mathrm{a})}$ & $\mathrm{Ol}^{\mathrm{b})}$ & $\mathrm{Pig}^{\mathrm{a})}$ & Pig $^{\text {b) }}$ & Aug $^{\text {b) }}$ & $\operatorname{Aug}^{\text {a) }}$ & Aug $^{\text {b) }}$ & $\mathrm{Gl}^{\mathrm{a})}$ & $\mathrm{Si}^{\mathrm{a})}$ \\
\hline $\mathrm{SiO}_{2}$ & n.d. & n.d. & 37.5 & 37.3 & 52.4 & 53.6 & 52.4 & 52.4 & 55.6 & 60.2 & 0.08 & 38.8 & 38.6 & 55.5 & 55.0 & 52.3 & 49.1 & 46.2 & 69.9 & 92.9 \\
\hline $\mathrm{TiO}_{2}$ & 15.8 & 52.9 & n.d. & n.d. & 0.62 & 0.48 & 1.30 & 0.98 & 0.12 & 0.15 & 1.26 & n.d. & 0.06 & 0.14 & 0.16 & 0.45 & 1.68 & 3.49 & 0.46 & 0.32 \\
\hline $\mathrm{Al}_{2} \mathrm{O}_{3}$ & 5.65 & n.d. & n.d. & n.d. & 1.71 & 0.98 & 1.61 & 1.13 & 27.5 & 24.6 & 7.36 & n.d. & n.d. & 0.55 & 0.59 & 2.14 & 5.28 & 10.8 & 20.2 & 4.08 \\
\hline $\mathrm{Cr}_{2} \mathrm{O}_{3}$ & 26.3 & 1.15 & n.d. & n.d. & 0.75 & 0.38 & 0.78 & 0.42 & n.d. & n.d. & 55.7 & n.d. & 0.07 & 0.46 & 0.40 & 0.75 & 0.29 & n.d. & n.d. & n.d. \\
\hline $\mathrm{FeO}$ & 46.0 & 39.1 & 27.6 & 28.1 & 16.4 & 16.5 & 10.0 & 14.1 & 0.45 & 0.39 & 28.4 & 22.4 & 23.0 & 14.5 & 14.3 & 8.89 & 7.68 & 12.8 & 1.51 & 0.25 \\
\hline $\mathrm{MnO}$ & 0.72 & 0.78 & 0.54 & 0.58 & 0.57 & 0.62 & 0.44 & 0.54 & n.d. & n.d. & 0.62 & 0.47 & 0.41 & 0.48 & 0.54 & 0.41 & 0.21 & 0.32 & 0.10 & n.d. \\
\hline $\mathrm{MgO}$ & 3.64 & 4.96 & 34.1 & 34.0 & 21.9 & 22.3 & 16.6 & 19.2 & 0.14 & n.d. & 5.00 & 36.0 & 37.4 & 26.2 & 24.4 & 16.5 & 11.6 & 5.74 & 0.46 & 0.06 \\
\hline $\mathrm{CaO}$ & n.d. & n.d. & 0.12 & 0.16 & 4.77 & 4.79 & 16.4 & 10.1 & 10.4 & 5.88 & 0.05 & 0.15 & 0.16 & 2.71 & 4.65 & 17.1 & 22.0 & 21.0 & 3.83 & 0.79 \\
\hline $\mathrm{Na}_{2} \mathrm{O}$ & n.d. & n.d. & n.d. & n.d. & 0.15 & 0.11 & 0.34 & 0.19 & 5.15 & 7.09 & n.d. & n.d. & n.d. & 0.05 & 0.07 & 0.26 & 0.30 & 0.31 & 3.46 & 0.81 \\
\hline $\mathrm{K}_{2} \mathrm{O}$ & n.d. & n.d. & n.d. & n.d. & n.d. & n.d. & n.d. & n.d. & 0.42 & 1.25 & n.d. & n.d. & n.d. & n.d. & n.d. & n.d. & n.d. & n.d. & 0.21 & 0.05 \\
\hline 总和 & 98.1 & 99.0 & 100.0 & 100.2 & 99.3 & 99.7 & 99.8 & 99.0 & 99.8 & 99.7 & 98.6 & 98.0 & 99.8 & 100.7 & 100.2 & 98.9 & 98.2 & 100.8 & 100.1 & 99.2 \\
\hline $\mathrm{Fa} / \mathrm{Fs} / \mathrm{An}$ & & & 31.2 & 31.7 & 26.7 & 26.5 & 16.5 & 23.0 & 51.4 & 29.1 & & 25.8 & 25.6 & 22.4 & 22.5 & 14.7 & 13.6 & 25.6 & & \\
\hline $\mathrm{Fo} / \mathrm{Wo} / \mathrm{Or}$ & & & 68.8 & 68.3 & 9.9 & 9.9 & 34.7 & 21.1 & 2.5 & 7.4 & & & & 5.4 & 9.3 & 36.4 & 49.8 & 53.9 & & \\
\hline
\end{tabular}

*) Chm, 铬铁矿; Ilm, 钛铁矿; Ol, 橄榄石; Pig, 易变辉石; Aug, 普通辉石; Pl, 长石; $\mathrm{Si}$, 石英; Gl, 长石质玻璃. a) 核部; b) 边部; n.d. 低于检测限
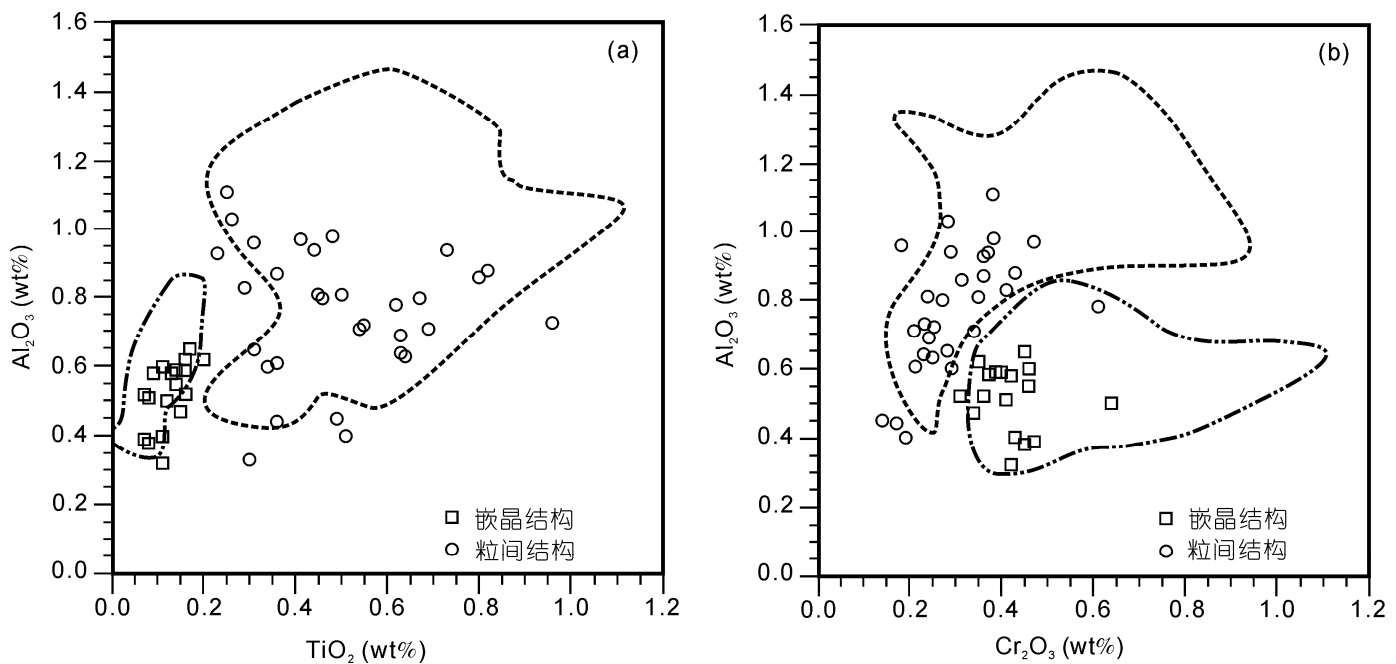

图 8 Y984028 与 GRV 99027 易变辉石的次要元素比较

(a) $\mathrm{Y} 984028$ 的主晶易变辉石 $\mathrm{TiO}_{2}$ 含量较低, 明显不同于粒间结构的易变辉石, $\mathrm{Al}_{2} \mathrm{O}_{3}$ 与 $\mathrm{TiO}_{2}$ 含量呈正相关. (b) $\mathrm{Y} 984028$ 中易变辉石的 $\mathrm{Al}_{2} \mathrm{O}_{3}$ 与 $\mathrm{Cr}_{2} \mathrm{O}_{3}$ 也与岩石结构相关, 两者有明显不同. 虚线圈出区域为 GRV 99027 的成分分布范围 ${ }^{[13]}$ 
$0.83 \pm 0.35 \mathrm{wt} \%, 0.54 \pm 0.29 \mathrm{wt} \%$ 和 $4.41 \pm 0.82 \mathrm{wt} \%$ (嵌晶区域). 相比, 2 种结构中的 $\mathrm{Cr}_{2} \mathrm{O}_{3}$ 含量几乎相同 (0.12 wt \% 1.01 wt \%). 另外, 嵌晶区域的 $\mathrm{Al}_{2} \mathrm{O}_{3}$ 含量 与 $\mathrm{CaO}$ 和 $\mathrm{TiO}_{2}$ 有正相关关系, 而粒间区域不具有这 种相关性(图 8). 从单个辉石颗粒的核部与边部成分 看, 嵌晶区域与粒间区域的易变辉石均没有明显的 环带特征(表 2), 它们的 $\mathrm{FeO} / \mathrm{MnO}$ 质量百分比为 27.8 \pm 2.3 . 代表性电子探针成分见表 2 .

普通辉石：普通辉石主要产出于粒间区域和嵌 晶区域的易变辉石边沿. 与易变辉石不同, 粒间区域 与嵌晶区域的普通辉石成分几乎一样(图 7), 普通辉 石的成分可从 $\mathrm{En}_{42.6} \mathrm{Fs}_{13.1} \mathrm{Wo}_{44.2}$ 变化至 $\mathrm{En}_{55.9} \mathrm{Fs}_{23.0^{-}}$ $\mathrm{Wo}_{21.1}$. 普通辉石中的次要元素主要有 $\mathrm{Al}_{2} \mathrm{O}_{3}(0.71$ wt \% 5.73 wt $\%), \mathrm{TiO}_{2}(0.30$ wt $\% \sim 1.90$ wt $\%), \mathrm{Cr}_{2} \mathrm{O}_{3}$ (0.09 wt \% 0.95 wt \%)和 $\mathrm{MnO}(0.27 \mathrm{wt} \% \sim 9.56 \mathrm{wt} \%)$. $\mathrm{Al}_{2} \mathrm{O}_{3}$ 和 $\mathrm{Cr}_{2} \mathrm{O}_{3}$ 的含量与 $\mathrm{CaO}(0.11 \mathrm{wt} \% \sim 19.64 \mathrm{wt} \%)$ 含量呈正相关. 普通辉石的 $\mathrm{FeO} / \mathrm{MnO}$ 质量百分比为 $25.2 \pm 2.4$. 代表性电子探针成分见表 2 .

长石: 在高对比度模式下，根据背散射电子图像 的亮度差异可以区分不同的长石颗粒(图 2). 定量分 析结果也说明明暗不同的长石具有成分差异, 亮灰 色的长石富 $\mathrm{CaO}$, 暗灰色的长石富 $\mathrm{K}_{2} \mathrm{O}$ (图 9). 电子 探针剖面分析结果表明, 明暗不同的长石颗粒成分 没有成分环带. 长石中含少量 $\mathrm{FeO}(0.17 \mathrm{wt} \% \sim 0.70$ $\mathrm{wt} \%$, 主要 $<0.60 \mathrm{wt} \%), \mathrm{TiO}_{2}(<0.26 \mathrm{wt} \%)$ 和 $\mathrm{MgO}$ $(<0.32 \mathrm{wt} \%)$. 富 $\mathrm{K}$ 长石 $\left(\mathrm{An}_{12.5} \mathrm{Ab}_{46.6} \mathrm{Or}_{40.8}\right)$ 仅在岩浆包 裹体中有产出. 代表性探针分析点见表 2 .

铬铁矿：粒间区域与嵌晶区域的铬铁矿有明显 不同的成分分布 (图 10). 嵌晶区域的铬铁矿贫 $\mathrm{TiO}_{2}(1.12 \mathrm{wt} \% \sim 1.93 \mathrm{wt} \%)$, 单颗粒成分均一, 且 $\mathrm{Cr}_{2} \mathrm{O}_{3}$ 和 $\mathrm{Al}_{2} \mathrm{O}_{3}$ 的原子比在 $\mathrm{Al}-\mathrm{Cr}-2 \mathrm{Ti}$ 三角图上沿 $\mathrm{Al}-\mathrm{Cr}$ 连线分布. 与嵌晶区域的铬铁矿相比, 粒间铬 铁矿成分较复杂, 其成分与产状有关. 当被大颗粒的 橄榄石包裹时, 铬铁矿的成分与嵌晶铬铁矿相似, 贫 $\mathrm{TiO}_{2}(1.07 \mathrm{wt} \%$ 3.17 wt \%), 但变化范围稍大. 粒间区 域的铬铁矿大部分都富 $\mathrm{TiO}_{2}(6.21 \mathrm{wt} \%$ 15.8 wt \%), $\mathrm{Cr} / \mathrm{Al}$ 原子比值近似为常数 $(\sim 4.1)$. 一些充填在粒间

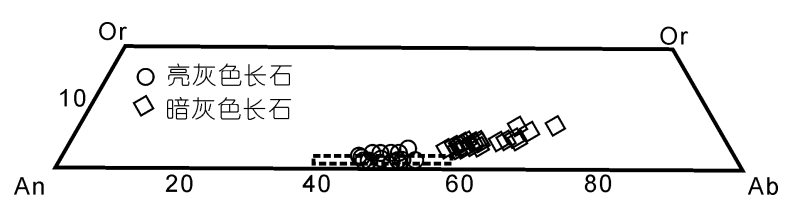

图9Y984028 与 GRV 99027(虚线区域[13])长石成分对比

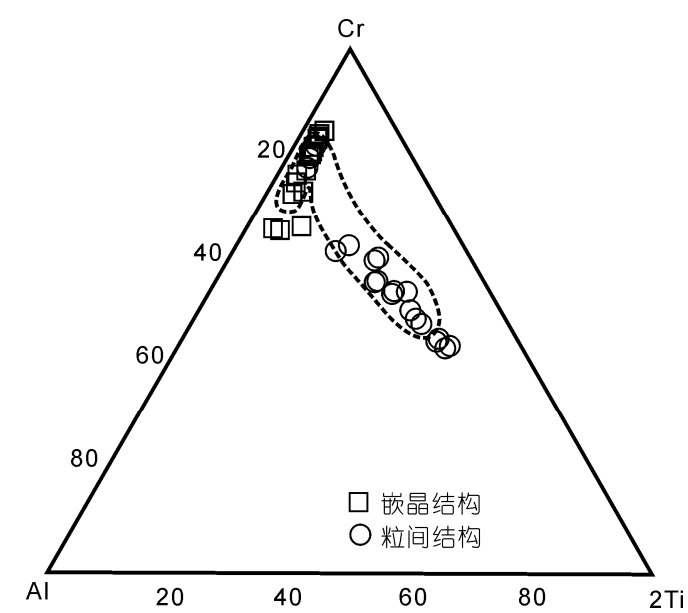

图 $10 Y 984028$ 中铬铁矿的成分

嵌晶铬铁矿贫 $\mathrm{TiO}_{2}$, 粒间铬铁矿富 $\mathrm{TiO}_{2}$, 两者 $\mathrm{Al}_{2} \mathrm{O}_{3}$ 含量差别很 小. 虚线圈出位置为 GRV 99027 的铬铁矿成分 ${ }^{[13]}$

的粗粒铬铁矿具有成分环带，核部贫 $\mathrm{TiO}_{2}(1.14 \mathrm{wt} \%$ $1.18 \mathrm{wt} \%)$. 当铬铁矿与钛铁矿伴生时, $\mathrm{TiO}_{2}$ 含量从铬 铁矿的核部(14.2 wt\% 15.3 wt\%)向钛铁矿方向递减 (10.4 wt\% 12.2 wt\%). 代表性铬铁矿电子探针成分 见表 2 .

钛铁矿：钛铁矿颗粒本身及不同颗粒之间的成 分都比较均一, 主要成分为 $\mathrm{TiO}_{2}(52.8 \pm 1.1 \mathrm{wt} \%)$ 和 $\mathrm{FeO}(39.3 \pm 0.9 \mathrm{wt} \%)$; 含有少量 $\mathrm{MgO}(4.33 \mathrm{wt} \%$ 5.90 wt $\%), \mathrm{MnO}(0.77 \mathrm{wt} \%$ 0.90 wt $\%)$ 和 $\mathrm{Cr}_{2} \mathrm{O}_{3}(0.48 \mathrm{wt} \%$ $1.42 \mathrm{wt} \%)$. 代表性电子探针成分可见表 2.

\section{3 讨论}

\section{1 与其他二辉橄榄岩质火星陨石的相似性}

Y984028 的岩石结构和矿物成分与 GRV 99027 非常相似. 它们都由两种不同的岩石结构组成：嵌晶 结构和粒间结构. 嵌晶结构主要由橄榄石客晶、易变 辉石主晶和自形铬铁矿包裹体组成; 粒间结构由自 形-半自形橄榄石、易变辉石、普通辉石和粒间充填 的长石玻璃组成，粒间常有白磷钙矿、钛铁矿、富 $\mathrm{Ti}$ 铬铁矿、磁黄铁矿和斜锆石产出. 另外, Y 984028 与 GRV 99027 中的矿物成分也非常接近. 矿物成分 均与产状有关，橄榄石和辉石在嵌晶区域相对贫 $\mathrm{FeO}$ ，铬铁矿相对贫 $\mathrm{TiO}_{2}$.

虽然二辉檄榄岩质火星陨石的岩石结构非常相 似，但模式含量变化较大(表 1), 可能与选样的不均 一性有关，如长石玻璃多产出于粒间区域。与其他二 
辉橄榄岩质火星陨石相比, Y984028 与 GRV 99027 在 粒间区域的长石玻璃模式含量均很低(表 1), 表明这 两个样品本来就贫长石玻璃. 虽然二辉橄榄岩质火 星陨石一般在嵌晶区域贫长石玻璃, 但 ALHA77005 和 LEW 88516 在嵌晶区域的的长石玻璃含量与 Y984028 和 GRV 99027 粒间区域的长石玻璃含量相 当(表 1). Y984028 嵌晶区域的辉石含量 (58.3 vol\%)明 显高于橄榄石的含量(39.6 vol\%), 与 GRV 99027 的 模式含量相似(67.8 vol\% vs. 30.9 vol\%). 相比, ALHA77005 和 LEW 88516 明显富橄榄石(表 1).

Y984028 的矿物成分与 GRV 99027 非常相似. Y984028 的橄榄石成分分布几乎与 GRV 99027 重叠 (图 6), 均有 2 个峰, 分别在 $\mathrm{Fa}_{27}$ 和 $\mathrm{Fa}_{30 \sim 31}$ 处. $\mathrm{Y} 984028$ 的辉石成分也落在 GRV 99027 的辉石成分变化范围 内(图 7 和 8). Y984028 与 GRV 99027 的铬铁矿成分 也非常接近(图 10). 两者长石玻璃的化学成分差异最 大, $\mathrm{Y} 984028$ 的长石 $\mathrm{CaO}$ 含量 $\left(\mathrm{An}_{23 \sim 55}\right)$ 变化明显大于 GRV 99027( $\left.\mathrm{An}_{42 \sim 61}\right)$ (图 9).

虽然 Y984028 的岩石结构和矿物成分与 GRV 99027 非常接近, 但 Y984028 不是 GRV 99027 的成对 陨石. Y984028 可见诸多冲击变质特征, 如长石玻璃 以及其他硅酸盐质熔体形成的流动结构、重结晶形成 细粒化的橄榄石和铬铁矿角砾等, 这些特征与 GRV 99027 和其他二辉橄榄岩质火星陨石明显不同. 一种 可能是, Y984028 与其他二辉橄榄岩质火星陨石来自 火星的同一岩浆房，在同一次撞击事件中离开母体. 或者, Y984028 来自火星的另一个位置另一次撞击事 件. 要证实以上推断, 需要开展 Y984028 的宇宙暴露 历史研究.

\section{2 卸压阶段的淬火特征}

与其他二辉橄榄岩质火星陨石相似，Y984028 也 经历过强烈的冲击变质作用, 其中的长石已经全部 转变成玻璃, 可见网状冲击熔脉. Y984028 中的长石 在正交偏光下为全消光, 表明该陨石遭受撞击后还 经历了微弱的热变质, 在细脉区域中可见光滑的辉 石玻璃也证明了这一点. 与之对比, GRV99027 在撞 击后, 经历的热变质较强, 其长石玻璃和熔融囊已经 高度重结晶 ${ }^{[13]}$. 在 ALH 77005 中也发现有长石玻 璃 ${ }^{[2,4]}$ 和熔融囊的部分重结晶现象 ${ }^{[24]}$.

Y984028 的熔脉非常特殊, 其中包含大量的常 温常压矿物相碎屑、长石玻璃及共生铁镁质硅酸盐熔
体普遍发育流动结构、广泛发育橄榄石和铬铁矿的细 粒化结构. 没有发现橄榄石、辉石和长石等矿物的高 压多形不可能是退变质所致, 因为 Y984028 在冲击 事件后, 仅遭受了轻微的热变质作用. 上述这些特征 表明, Y984028 在撞击过程中发生了部分熔融，在压 力峰值卸载后, 在较低压力下发生淬火. 如果熔脉在 高压下发生淬火, 那么橄榄石和辉石的高压相则可 以从熔体中结晶析出, 如普通球粒陨石中发现的大 量高压相矿物 ${ }^{[23]}$.

熔脉中产出大量的矿物碎屑表明撞击产生的温 度不足以使 Y984028 发生大范围熔融, 仅在局部发 生了较弱的部分熔融, 最后在熔脉中残留了大量的 矿物碎屑. 这些特征与普通球粒陨石有很大差异, 后 者的冲击熔脉中常残留粗粒浑圆状的围岩角砾. Y984028 很可能在冲击压力释放后发生了绝热熔融. 在 Y984028 的熔脉中, 大部分长石已经发生熔融, 其 次有部分辉石发生熔融, 橄榄石主要以矿物角砾的 形式散布在熔脉中, 不同矿物发生部分熔融的比例 为长石 $>$ 辉石 $>$ 橄榄石, 这一趋势可能与三者的熔点 相关, 在常压下的熔融温度为长石 (约 $1300 \sim 1400^{\circ} \mathrm{C}$ ) $<$ 辉石 $\left(\sim 1500^{\circ} \mathrm{C}\right)<$ 橄榄石 $\left(1900^{\circ} \mathrm{C}\right)$.

熔脉中橄榄石和铬铁矿发育细粒化结构表明 Y984028 最初开始熔融的温度接近橄榄石固相线, $\sim 1900{ }^{\circ} \mathrm{C}$, 该温度落在铬铁矿的熔融温度范围内 (约 $1850 \sim 2200^{\circ} \mathrm{C}$ ), 因而可以观察到橄榄石和铬铁矿的 边缘因重结晶形成细粒化结构. 由于辉石 $\left(\mathrm{Fs}_{15 \sim 27}\right)$ 的 熔融温度比橄榄石 $\left(\mathrm{Fa}_{29.6 ~ 33.5}\right)$ 的熔融温度低, 所以冲

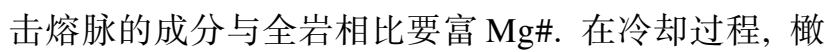
榄石先从熔体中结晶析出, 其成分与残留的橄榄石 相比明显贫 $\mathrm{FeO}$. 随着橄榄石结晶程度增加, 在熔体 中同时可能伴随有金属和硫化物的氧化过程, 熔体 中的 $\mathrm{Mg} \#$ 降低, 最后在残留橄榄石的边部形成富 $\mathrm{FeO}$ 的薄边, 形成图 4(d)中所示的环带结构.

另一种可能性是 Y984028 与高冲击强度的普通 球粒陨石相似，遭受强烈撞击形成熔脉，但是其冲击 事件的 P-T-t 历史较为特殊, 压力释放得较快而温度 一直相对较高, 因此其温压条件没有达到林伍德石、 镁铁榴石等高压矿物形成的范围. 然而, 依据该模型 应该在熔脉中可以观察到强烈的熔融特征，与 Y984028 熔脉中残留大量的不规则矿物碎屑相冲突. 另外, 冲击细脉 $(10 \mu \mathrm{m}$ 宽)的结构特征显示其在压力 卸载前就已经淬火, 然而在这些区域也没有发现高压 
矿物的产出, 这一点也与上述 P-T-t 演化过程不相符.

在冲击事件后, Y984028 基岩中的所有长石颗粒 已经转变成玻璃相, 在其他火星陨石也发现长石颗 粒在高温高压下熔融淬火形成的熔长石, 然而这两 者的形貌结构以及形成机制有明显的不同 ${ }^{[25]}$. 因为 Y984028 基岩中大多数长石玻璃颗粒仍然保留其原 始的形貌轮廓, 边界清晰平直, 并未出现明显熔融流 动结构; 长石玻璃内部非常均匀, 未混人橄榄石和辉 石碎屑或熔体. 此外, 共生的长石颗粒在背散射电子 图像上可见清晰的边界和亮度差异(图 2(a)和(b)), 说 明没有发生过熔融. 电子探针成分剖面分析结果表 明这些长石颗粒成分均匀, 没有出现环带. Y984028 中长石玻璃的岩石矿物学特征表明其并未经历过熔 融淬火的过程.

\section{4 结论}

Y984028 嵌晶区域和粒间区域的橄榄石、辉石和
铬铁矿的成分具有双峰分布, 嵌晶区域的橄榄石和 辉石贫铁, 铬铁矿贫钛, 而粒间区域的橄榄石和辉石 富铁, 铬铁矿富钛, 为典型的二辉橄榄岩质火星陨石 特征, 与 GRV 99027 具有非常相似的岩石学和矿物 学特征. 然而, Y984028 与 GRV 99027 在冲击特征上 有明显的差异, 表明两者不是成对陨石.

Y984028 遭受了强烈的冲击作用, 形成了冲击 熔脉, 并且基岩中的全部长石转变成了玻璃相. 正交 偏光下长石的全消光, 以及熔脉中玻璃相, 表明 Y984028 在主撞击事件后没有遭受明显的热变质. Y984028 冲击熔脉中包含大量不规则矿物碎屑, 橄 榄石和铬铁矿普遍发育细粒化结构, 但未转变成矿 物的高压相. 这些特征表明 Y984028 在撞击压力释 放中发生了绝热熔融, 然后在低压下淬火冷却. 在基 岩中, 组成不同的长石颗粒具有清晰边界, 且无明显 的成分环带特征，表明基岩中的长石玻璃没有经历 熔融, 而是固-固相变形成.

\section{参考文献}

1 Harvey R P, Wadhwa M, McSween Jr H Y, et al. Petrography, mineral chemistry, and petrogenesis of Antarctic shergottite LEW88516. Geochim Cosmochim Acta, 1993, 57: 4769-4783

2 Ikeda Y. Petrography and petrology of the ALH-77005 shergottite. Antarct Meteorite Res, 1994, 7: 9-29

3 Ikeda Y. Petrology and mineralogy of the Y-793605 Martian meteorite. Antarct Meteorite Res, 1997, 10: 13-40

4 Treiman A H, McKay G A, Bogard D D, et al. Comparison of the LEW88516 and ALHA77005 Martian meteorites: Similar but distinct. Meteoritics, 1994, 29: 581-592

5 Gleason J D, Kring D A, Hill D H, et al. Petrography and bulk chemistry of Martian lherzolite LEW88516. Geochim Cosmochim Acta, 1997, 61: 4007-4014

6 Mikouchi T, Miyamoto M. Yamato-793605: A new lherzolitic shergottite from the Japanese Antarctic meteorite collection. Antarct Meteorite Res, 1997, 10: 41-60

7 Borg L E, Nyquist L E, Wiesmann H, et al. Constraints on the petrogenesis of Martian meteorites from the Rb-Sr and Sm-Nd isotopic systematics of the lherzolitic shergottites ALH77005 and LEW88516. Geochim Cosmochim Acta, 2002, 66: 2037-2053

8 Morikawa N, Misawa K, Kondorosi G, et al. Rb-Sr isotopic systematics of lherzolitic shergottite Yamato-793605. Antarct Meteorite Res, 2001, 14: 47-60

9 Eugster O, Polnau E. Mars-Earth transfer time of lherzolite Yamato-793605. Antarct Meteorite Res, 1997, 10: 143-149

10 Eugster O, Weigel A, Polnau E. Ejection times of Martian meteorites. Geochim Cosmochim Acta, 1997, 61: 2749-2757

11 孔屏, Fabel D, Brown R, 等. 南极格罗夫山火星陨石 GRV 99027 的宇宙射线暴露年龄. 中国科学 D 辑: 地球科学, 2007, 37: 1020-1023

12 林杨挺, 王道德, 缪秉鬼, 等. 南极格罗夫山陨石 GRV 99027: 一个新的火星陨石. 科学通报, 2003, 48: 1806-1810

13 Lin Y T, Guan Y B, Wang D D, et al. Petrogenesis of the new lherzolitic shergottite Grove Mountains 99027: Constraints of petrography, mineral chemistry, and rare earth elements. Meteorit Planet Sci, 2005, 40: 1599-1619

14 Lin Y T, Qi L, Wang G Q, et al. Bulk chemical composition of lherzolitic shergottite Grove Mountains 99027-Constraints on the mantle of Mars. Meteorit Planet Sci, 2008, 43: 1179-1187

15 Hsu W B, Guan Y B, Wang H N, et al. The lherzolitic shergottite Grove Mountains 99027: Rare earth element geochemistry. Meteorit Planet Sci, 2004, 39: 701-709

16 Mikouchi T, Kurihara T. Mineralogy and petrology of paired lherzolitic shergottites Yamato 000027, Yamato 000047, and Yamato 000097: Another fragment from a Martian "lherzolite" block. Polar Sci, 2008, 2: 175-194 
17 Gillet P, Barrat J A, Beck P, et al. Petrology, geochemistry, and cosmic-ray exposure age of lherzolitic shergottite Northwest Africa 1950. Meteorit Planet Sci, 2005, 40: 1175-1184

18 Miao B, Ouyang Z, Wang D, et al. A new Martian meteorite from Antarctica: Grove Mountains (GRV) 020090. Acta Geol Sin, 2004, 78: 1034-1041

19 Mikouchi T, Kurihara T, Miyamoto M. Petrology and mineralogy of RBT 04262: Implications for stratigraphy of the lherzolitic shergottite igneous block. In: LPS XXXIX. League City, Texas: National Aeronautics and Space Administration, 2008. 2403

20 Usui T, Sanborn M E, Wadhwa M, et al. Petrogenesis of geochemically enriched lherzolitic shergottites RBT 04261 and RBT 04262. In: 71st Annual Meteoritical Society Meeting. Matsue: National Aeronautics and Space Administration, 2008, 43(Suppl): 5052

21 Hu S, Feng L, Lin Y T. Petrology and mineral chemistry of the Y984028 lherzolitic shergottite. In: Antarct Meteorit Symp XXXII. Tokyo: National Institute of Polar Research, 2009. 22-23

22 Delaney J S. Petrological comparison of LEW 88516 and ALHA 77005 shergottites. Meteoritics, 1992, 27: 213-214

23 Chen M, Sharp T, El Goresy A, et al. The majorite-pyrope + magnesiowustite assemblage: Constraints on the history of shock veins in chondrites. Science, 1996, 271: 1570-1573

24 Walton E L, Herd C D K. Dynamic crystallization of shock melts in Allan Hills 77005: Implications for melt pocket formation in Martian meteorites. Geochim Cosmochim Acta, 2007, 71: 5267-5285

25 El Goresy A, Miyahara M, Ozawa S, et al. Liquidus high-pressure assemblages in shocked martian shergottites: Constraints to equilibrium peak shock-pressures and consequences to radiometric ages. In: 73rd Annual Meteoritical Society Meeting. New York: National Aeronautics and Space Administration, 2010. 5020 